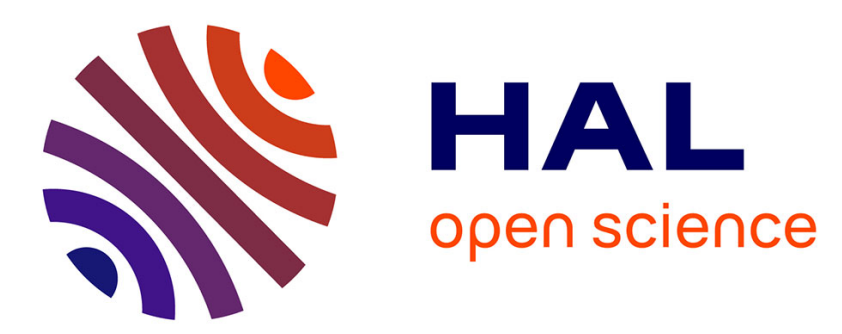

\title{
Real versus synthesized fractal surfaces: Contact mechanics and transport properties
}

Christophe Vallet, Didier Lasseux, Philippe Sainsot, H. Zahouani

\section{To cite this version:}

Christophe Vallet, Didier Lasseux, Philippe Sainsot, H. Zahouani. Real versus synthesized fractal surfaces: Contact mechanics and transport properties. Tribology International, 2008, 42 (2), pp.250259. 10.1016/j.triboint.2008.06.005 . hal-00413237

\section{HAL Id: hal-00413237 https://hal.science/hal-00413237}

Submitted on 3 Sep 2009

HAL is a multi-disciplinary open access archive for the deposit and dissemination of scientific research documents, whether they are published or not. The documents may come from teaching and research institutions in France or abroad, or from public or private research centers.
L'archive ouverte pluridisciplinaire HAL, est destinée au dépôt et à la diffusion de documents scientifiques de niveau recherche, publiés ou non, émanant des établissements d'enseignement et de recherche français ou étrangers, des laboratoires publics ou privés. 


\title{
Real versus synthesized fractal surfaces: contact mechanics and transport properties
}

\author{
C. Vallet ${ }^{*, a}$, D. Lasseux ${ }^{*, b}$, P. Sainsot ${ }^{c}$, H. Zahouani ${ }^{\mathrm{d}}$ \\ ${ }^{a}$ EDF RED, avenue des Renardières - Ecuelles, 77818 Moret-sur-Loing Cedex, \\ France \\ ${ }^{\mathrm{b}}$ TREFLE, CNRS UMR8508, Esplanade des Arts et Métiers, 33405 Talence \\ Cedex, France \\ ${ }^{\mathrm{c}}$ LaMCoS, INSA-Lyon, CNRS UMR5259, F69621, France \\ ${ }^{\mathrm{d}}$ ENISE, 58 rue Jean Parot, 42000 Saint Etienne, France
}

\begin{abstract}
In this work, we analyze diffusion and viscous flow through the aperture field of a contact between rough surfaces pressed against each other. The purpose of our study is to validate the use of synthesized rough surfaces instead of real ones for surfaces exhibiting fractal properties. Models for mechanical deformation of asperities as well as for transport resulting from a pressure gradient or a species concentration gradient are presented. At the macroscopic scale, viscous and diffusive transports only depend on transmissivity $\mathbf{K}$ and diffusivity $\mathbf{D}$ respectively. Both tensors $\mathbf{K}$ and $\mathbf{D}$ are intrinsic, which means they can be entirely determined from the aperture field. Two kinds of surfaces obtained from two different machining processes lapping and sand-blasting - are considered. The dependence of the global contact area, distribution of local contact areas, $\mathbf{K}$ and $\mathbf{D}$ upon the average contact pressure is compared for real surfaces and their analogue synthesized surfaces. The comparison over a wide range of contact pressures leads to the conclusion that a fractal representation is a robust representation for the contact mechanics as well as for transport properties for this class of surfaces. This validates the overall procedure described in this work.
\end{abstract}

Key words: Self-affine fractal surfaces, Rough contact, Transport properties

\footnotetext{
* Corresponding author

Email addresses: christophe.vallet@edf.fr (C. Vallet), didier.lasseux@bordeaux.ensam.fr (D. Lasseux).
} 


\section{Introduction}

Solid surfaces, whatever the machining process, contain irregularities or deviations from the ideal geometrical form [1]-[2]. Moreover, irregularities occur at many scales, varying from body dimensions to interatomic distances. No machining method, however precise, can produce an ideally smooth and flat surface. Hence, when two surfaces are pressed against each other, contact is not complete but occurs at very localized spots. Determination of transport properties of this connected aperture field, i.e. parameters relating the fluid flux to the driving force, is of great importance in many industrial applications. Amongst others, we can cite sealing in mechanical industry and more particularly in the design and safety of nuclear power plants or cryotechnic rocket engines, where the sealing of some units is performed by direct contact between metallic surfaces [3].

In this paper, we present a deterministic approach to estimate the transport properties of a rough contact from initial roughnesses. When surfaces are pressed against each other, roughnesses deform under the action of the applied load. As a consequence, transport properties of a rough contact are expected to strongly depend on the aperture field modified by surface flattening. To estimate these transport properties, we propose a three step-procedure which consists in: i) starting from an initial measured or synthetic surface roughness, ii) computing surface deformations and iii) computing flow through the rough contact. Here, this procedure is restricted to small scale defaults, which means that only microroughness is considered, eliminating form errors and waviness appearing at larger scales. Computations are thus performed on small surfaces, large enough, however, to be representative of microroughness. These surface elements are thus considered as the periodic unit cell of an infinite surface. Moreover, we assume that the two contacting surfaces remain parallel and that a uniform load is applied so that only normal stresses are considered.

Our analysis is focused on rough surfaces, whose texture exhibits fractal properties. As shown in many references in the literature [4-8], several machining processes lead to surfaces having this kind of properties. In particular, Majumdar and Bhushan [7] reported that processes producing deterministic texture do not yield self-affine fractal surfaces, whereas those producing random texture do. Self-affine surfaces can theoretically be characterized and synthesized using only two scale-independent parameters extracted from surface topology and referred to as the fractal dimension $D_{f}$ and scale constant $C$ [4]. Within this context, the objective of the present work is to validate the use of synthetic self-affine surfaces as the initial step i) of the above mentioned procedure to estimate transport properties of the contact. This would avoid using real surfaces that require machining and detailed roughness measurement. In fact, the use of synthetic surfaces represent a major advantage if the aim is to perform 
a parametric analysis of the behavior of self-affine surfaces in a contact in order to aid to their design. To achieve this validation, a thorough comparison of contact areas as well as viscous and diffusive transport properties obtained from the above mentioned computational procedure is performed between two real surfaces and their analogue synthesized ones. This is performed for an apparent contact pressure ranging from 7 to $600 \mathrm{MPa}$. The two real surfaces under consideration are obtained by lapping and sand-blasting respectively. Lapping is a machining operation that consists in rubbing two surfaces with an abrasive in between, while sand-blasting consists in projecting at high speeds a stream of solid particles on the surface.

In section 2 of this paper, we present an approach to determine fractal properties of isotropic surfaces. This approach is applied to a real lapped surface and to a real sand-blasted surface. Finally, equivalent self-affine fractal surfaces are synthesized using a Fourier filtering method [9].

In section 3, we first present an elasto-plastic deformation model which allows the determination of surface deflections caused by tightening. In a second step, we describe fluid flow models through a rough contact due to viscous and diffusive effects. The macroscopic viscous flow model introduces a transmissivity tensor $\mathbf{K}$ which linearly relates the viscous flow rate per unit width of the contact to the macroscopic pressure gradient in the fluid. Similarly, the macroscopic diffusive model introduces a diffusivity tensor $\mathbf{D}$ which linearly relates the diffusive flow rate per unit width of the contact to the macroscopic species gradient. We show that the two tensors $\mathbf{K}$ and $\mathbf{D}$ are intrinsic, i.e. they only depend on the aperture field $h$.

Section 4 is dedicated to the results. To simplify, although models presented in section 3 allow the treatment of a contact between two rough surfaces, the contact between a rough surface pressed against a perfectly rigid and smooth plane is considered. Results obtained from real surfaces are compared to those obtained from equivalent synthesized surfaces. This comparison is performed on the distributions of contact spots on the one hand, and on the transport properties of the rough contact on the other.

\section{Analysis and simulation of isotropic self-affine fractal surfaces}

Self-similarity refers to fractal objects that are equally magnified in all directions. However, many objects in nature, like machined surface, are rather self-affine, which means that they have unequal scaling magnification in different directions [10]. The power spectral density $P\left(\omega_{x}, \omega_{y}\right)$ of an isotropic 
self-affine surface $z(x, y)$ follows a power law behavior given by [9]:

$$
P\left(\omega_{x}, \omega_{y}\right)=\lim _{L_{x}, L_{y} \rightarrow \infty} \frac{1}{L_{x} L_{y}}\left|\int_{0}^{L_{x} L_{y}} \int_{0}^{z} z(x, y) e^{-\mathrm{i}\left(x \omega_{x}+y \omega_{y}\right)} d x d y\right|^{2}=\frac{C^{2 D_{f}-4}}{\omega_{e q}^{8-2 D_{f}}}
$$

where

$$
\omega_{e q}=\sqrt{\omega_{x}^{2}+\omega_{y}^{2}}
$$

For a surface, $D_{f}$ must verify:

$$
2 \leq D_{f} \leq 3
$$

Equation (1) is equivalent to:

$$
\left|\widetilde{z}\left(\omega_{x}, \omega_{y}\right)\right| \propto \frac{C^{D_{f}-2}}{\omega_{e q}^{4-D_{f}}}
$$

where $\widetilde{z}\left(\omega_{x}, \omega_{y}\right)$ are the Fourier coefficients of $z(x, y)$ :

$$
\widetilde{z}\left(\omega_{x}, \omega_{y}\right)=\int_{-\infty}^{+\infty} \int_{-\infty}^{+\infty} z(x, y) e^{-\mathrm{i}\left(x \cdot \omega_{x}+y \cdot \omega_{y}\right)} d x d y
$$

From equations (1) or (4), it is clear that a self-affine fractal surface can be characterized by only two parameters: the fractal dimension $D_{f}$, which can be estimated from the slope of $\log P=f\left(\log \omega_{e q}\right)$, and a scale factor $C$ which can be determined from the intercept point on the same graph. The dimension of $C$ is length. On the one hand, $D_{f}$ is related to the relative power of the frequency content and is an indicator of the degree of irregularity of the surface such that the larger $D_{f}$ is, the more irregular the surface. On the other hand, $C$ is related to the amplitude of all frequencies. This means that $C$ is connected to roughness amplitude parameters such as $R a$, the arithmetic mean of the absolute values of $z$, or the root mean square $(\mathrm{rms})$ of $z$. These two parameters $D_{f}$ and $C$ are theoretically scale-independent. However, for real surfaces, it appears that when changing the lateral resolution of the measuring instrument, a lateral shift is observed in the power function plots, whereas their slopes remain constant [4]. This indicates that in practice, if $D_{f}$ is unique and scale-independent, $C$ is not. In the following, we therefore chose to characterize a self-affine fractal surface by its fractal dimension $D_{f}$, and for completeness, the roughness amplitude parameter $R a$, which also depends on the size of the surface, is used instead of $C$. This choice is motivated by the fact that it is a more physical parameter, directly accessible from measurement and widely used for engineering purposes.

In this section, the fractal nature of a lapped surface and of a sand-blasted surface is investigated. The lapped surface was finished with abrasive particles 
of about $1 \mu \mathrm{m}$ size while the sand-blasted surface was machined by streams of microbeads of $70-110 \mu \mathrm{m}$ size. The roughnesses of these surfaces were measured by white light interferometry on machined parts. Measurement parameters (surface dimensions, number of points, sampling intervals) are given in table 1. Surface dimensions $L_{x}, L_{y}$ are assumed to be large enough and sampling intervals $\Delta_{x}=L_{x} / n_{x}, \Delta_{y}=L_{y} / n_{y}$ small enough to be representative of the microroughness. First, fractal properties of these textures are analyzed and then equivalent self-affine fractal surfaces are synthesized.

Table 1

Surface parameters -

\begin{tabular}{ccccc}
\hline Surface & Dimensions & Sampling & $R a$ & $D_{f}$ \\
& $L_{x} \times L_{y}$ & $n_{x} \times n_{y}$ & & \\
\hline lapped & $462 \times 607 \mu m$ & $480 \times 736 p t s$ & $\mathbf{0 . 4} \mu m$ & $\mathbf{2 . 5 0}$ \\
sand-blasted & & & $\mathbf{1 . 0 \mu m}$ & $\mathbf{2 . 5 1}$ \\
\hline
\end{tabular}

In figure 1a, we have represented the power spectral density $P$ of the real lapped surface versus the equivalent angular frequency $\omega_{e q}$. Whereas some dispersion is observed, it appears that this surface exhibits fractal properties for $\omega_{e q}$ larger than a cut-off frequency $\omega_{\min }$, i.e. for wavelengths lower than $\frac{2 \pi}{\omega_{\min }}=20 \mu \mathrm{m}$. For $\omega_{e q}<\omega_{\min }$, it will be assumed that $P$ is constant (see [11]). The theoretical profile of $P$ is fitted in the least squares sense, allowing the identification of $D_{f}$ and $\omega_{\min }$. Similarly, the graph in figure 2a indicates that the real sand-blasted surface has a fractal trend for all frequencies investigated. These two surfaces have almost the same fractal dimension $D_{f}$ (see table 1 ), but since they have been machined with particles of different sizes, they are not fractal at the same scales. They also exhibit significant different roughness amplitudes as indicated by $R a$ values.

The approach to synthesize a self-affine fractal surface is based on the "Fourier filtering method" [9] which enables to generate a correlated random pattern. This method consists in generating a Gaussian white noise $a_{m n}\left(1 \leq m \leq n_{x}\right.$ and $\left.1 \leq n \leq n_{y}\right)$ in the physical space. Defining $\tilde{a}_{k l}$ as the discrete Fourier transform of $a_{m n}$, Fourier coefficients $\tilde{z}_{k l}$ can then be deduced by introducing a correlation among $\tilde{a}_{k l}$ such that the modulus of $\widetilde{z}_{k l}$ follows a power law as in equation (4). This transformation is given by:

$$
\begin{aligned}
& \tilde{z}_{00}=0 \\
& \tilde{z}_{k l}=\frac{\tilde{a}_{k l}}{\left(\omega_{k l}\right)^{4-D_{f}}}, \quad \forall k \in\left[0, \frac{n_{x}}{2}\right], \forall l \in\left[0, \frac{n_{y}}{2}\right]
\end{aligned}
$$




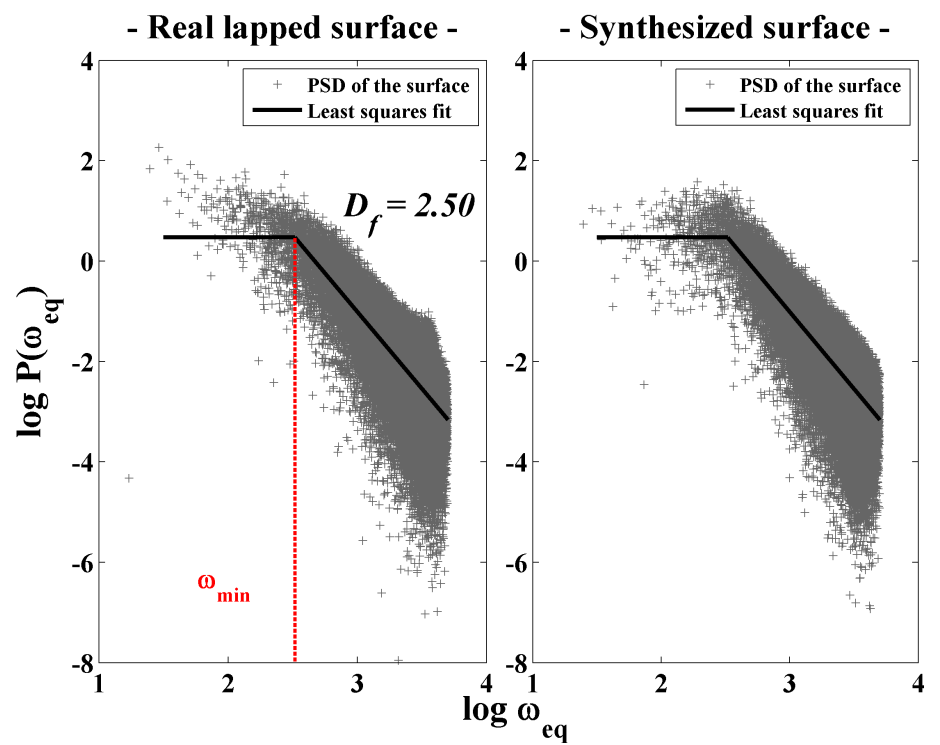

Fig. 1. Power spectral densities (PSD) of a) a real lapped surface and b) its equivalent synthesized surface -

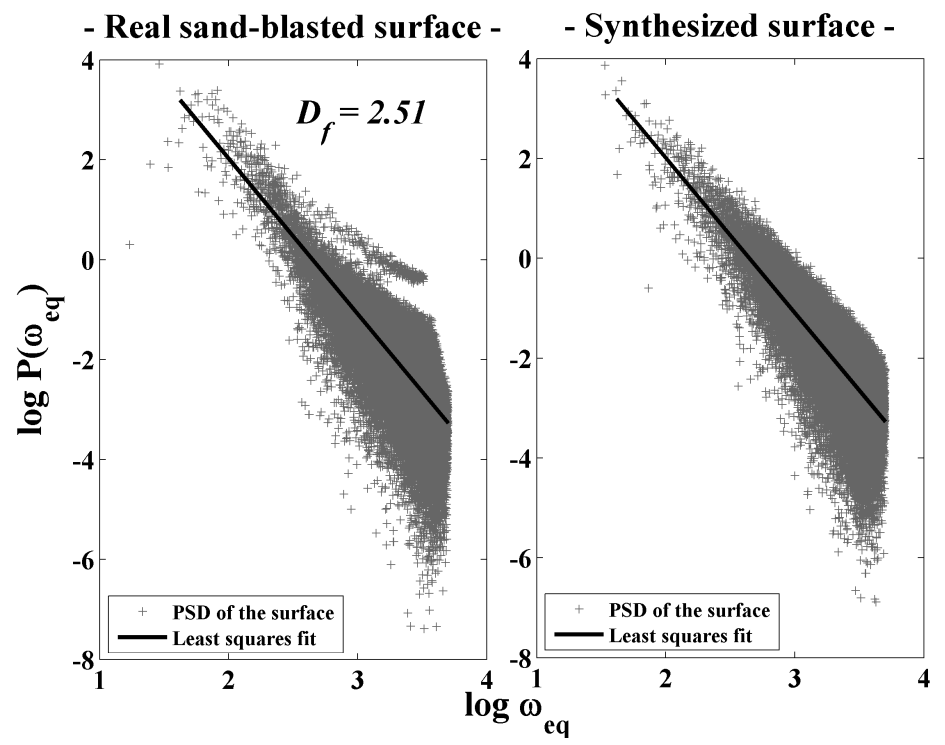

Fig. 2. Power spectral densities (PSD) of a) a real sand-blasted surface and b) its equivalent synthesized surface -

$$
\begin{array}{ll}
\tilde{z}_{k l}=\frac{\tilde{a}_{k l}}{\left(\omega_{n_{x}-k, l}\right)^{4-D_{f}}}, \quad \forall k \in\left[\frac{n_{x}}{2}+1, n_{x}-1\right] & \\
\quad, \forall l \in\left[1, \frac{n_{y}}{2}-1\right]
\end{array}
$$

where $\omega_{k l}=2 \pi \sqrt{\left(\frac{k}{L_{x}}\right)^{2}+\left(\frac{l}{L_{y}}\right)^{2}}$ (in $\left.\mathrm{rad} / \mathrm{mm}\right), L_{x}$ and $L_{y}$ being the surface dimensions in two orthogonal $x$ and $y$ directions respectively. Equation (6) ensures a zero mean of $z$. 
Since $\tilde{z}$ is a Fourier transform of a real sequence $z$, the remaining coefficients $\tilde{z}_{k l}$ are deduced as follows:

$$
\begin{aligned}
& \left\{\begin{array}{c}
\widetilde{z}_{0, l}=\operatorname{conj}\left(\widetilde{z}_{0, n_{y}-l}\right) \\
\widetilde{z}_{n_{x} / 2, l}=\operatorname{conj}\left(\widetilde{z}_{n_{x} / 2, n_{y}-l}\right)
\end{array}, \forall l \in\left[\frac{n_{y}}{2}+1, n_{y}-1\right]\right. \\
& \left\{\begin{array}{c}
\widetilde{z}_{k, 0}=\operatorname{conj}\left(\widetilde{z}_{n_{x}-k, 0}\right) \\
\widetilde{z}_{k, n_{y} / 2}=\operatorname{conj}\left(\widetilde{z}_{n_{x}-k, n_{y} / 2}\right)
\end{array}, \forall k \in\left[\frac{n_{x}}{2}+1, n_{x}-1\right]\right. \\
& \widetilde{z}_{k l}=\operatorname{conj}\left(\widetilde{z}_{n_{x}-k, n_{y}-l}\right), \quad \forall k \in\left[\frac{n_{x}}{2}+1, n_{x}-1\right], \forall l \in\left[\frac{n_{y}}{2}+1, n_{y}-1\right] \\
& \widetilde{z}_{k l}=\operatorname{conj}\left(\widetilde{z}_{n_{x}-k, n_{y}-l}\right), \quad \forall k \in\left[1, \frac{n_{x}}{2}-1\right], \forall l \in\left[\frac{n_{y}}{2}+1, n_{y}-1\right]
\end{aligned}
$$

where $\operatorname{conj}(\varphi)$ is the complex conjugate of $\varphi$.

The self-affine fractal surface $z_{m n}$ is then obtained by a discrete inverse Fourier transform of $\tilde{z}_{k l}$ :

$$
z_{m n}=\frac{1}{n_{x} n_{y}} \sum_{k=0}^{n_{x}-1 n_{y}-1} \sum_{l=0} \tilde{z}_{k l} e^{\left(\mathrm{i} k \frac{2 m \pi}{n_{x}}+\mathrm{i} l \frac{2 n \pi}{n_{y}}\right)}
$$

and rescaled to obtain the desired roughness amplitude parameter $R a$ :

$$
\bar{z}_{m n}=\frac{R a}{\frac{1}{n_{x} n_{y}} \sum_{m=1}^{n_{x}} \sum_{n=1}^{n_{y}}\left|z_{m n}\right|} z_{m n}
$$

With this method, self-affine fractal surfaces were synthesized with the same parameters as those of real surfaces (see table 1). Power spectral densities of these synthesized surfaces were then computed and are reported in figures $1 \mathrm{~b}$ and $2 \mathrm{~b}$. To synthesize the equivalent lapped surface, the cut-off angular frequency $\omega_{\min }$ was also considered in addition to the two parameters $D_{f}$ and $R a$. Textures of the real and synthesized lapped surfaces are represented in figure 3, while those of the sand-blasted surfaces are reported in figure 4 . It appears that the textures of the lapped surfaces are more regular than those of the sand-blasted surfaces. This can be explained simply by the existence of a cut-off frequency $\omega_{\min }$ in the power spectral density of the lapped surface (see figures $1 \mathrm{a}$ and $1 \mathrm{~b}$ ). This cut-off fades away long wavelength roughnesses leading to a texture smoother than those of the sand-blasted surfaces. 


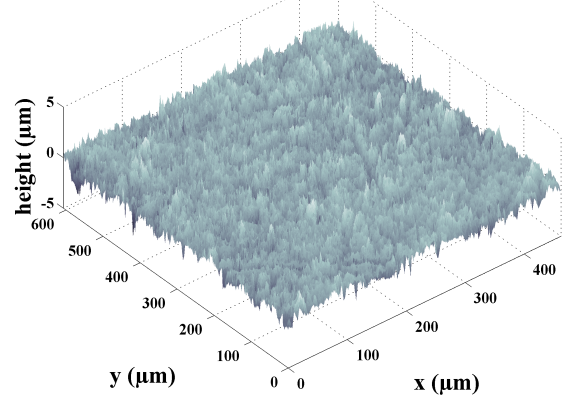

- Real lapped surface -

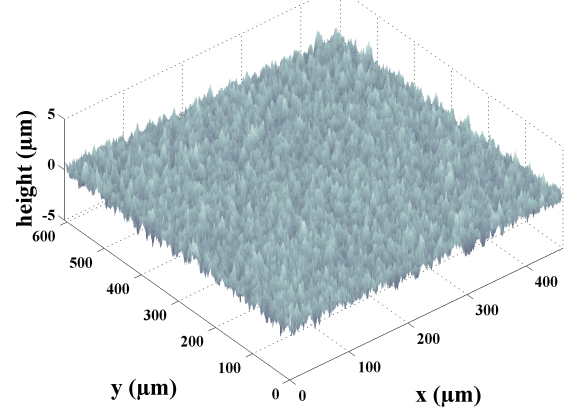

- Synthesized lapped surface -

Fig. 3. Textures of the lapped surfaces -

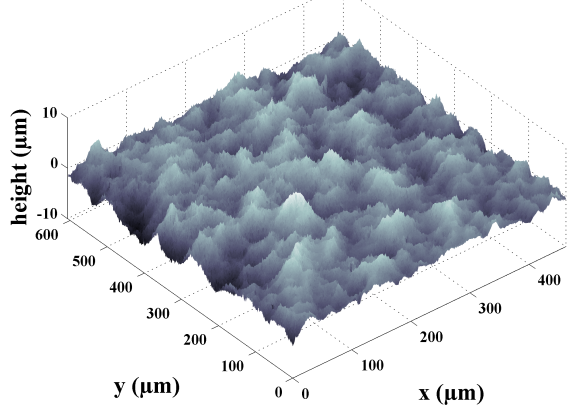

- Real sand-blasted surface -

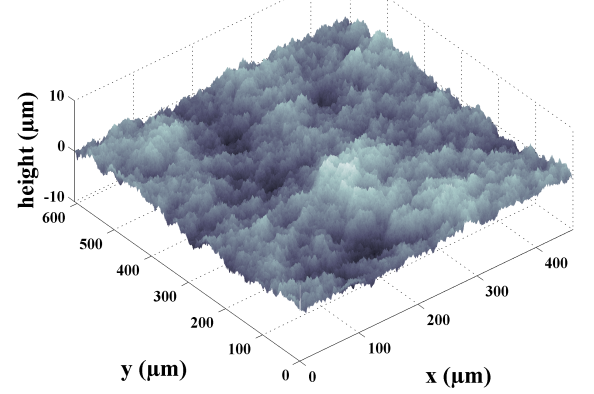

- Synthesized sand-blasted surface -

Fig. 4. Textures of the sand-blasted surfaces -

\section{Deformation and transport models}

\subsection{Elasto-plastic deformation model}

When two rough surfaces are brought in contact, effective contact only occurs on the top of asperities, which deform under the action of the applied load. During the past decades, the problem of the contact of rough surfaces has concentrated significant research efforts and a review of the main theories developed can be found in [1]. The major difficulty in modeling this problem lies in the adequate and precise enough description of the complete geometry of the rough contact, which, if a deterministic approach is chosen, may require a tremendous amount of data. Historically, stochastic models have been preferred to reduce computational times. The most widely used model is the model proposed by Greenwood and Williamson [12]. In this model, estimation of the true contact area and maximal contact pressure of a rough contact relies on the assumption that the rough surface is composed of spherical asperities 
having identical radii of curvature and heights following a Gaussian distribution about a mean plane. Many authors improved this model to include for instance, plastic deformations [13]-[14], anisotropic surfaces [15], asperities having elliptical paraboloid shapes [16]. However, the adequacy of these models is questionable since assumptions made on asperities are most of the time unrealistic and since statistical roughness parameters are not unique but strongly dependent on the resolution of the measuring instrument. With the expansion of computer capabilities, deterministic models have been developed. In that case, computations are directly performed on digitized surfaces, with no assumption on height distribution. In the present work, we adopted an elasto-plastic deformation model allowing us to take full account of interactions between all contact points and to predict contact geometry of rough surfaces under load. The problem is treated while supposing normal effects only. If the contact area is small compared to body dimensions and if slopes of asperities are small everywhere, solids in contact can be considered as semi-infinite. For the two real surfaces under consideration and with sampling parameters reported in table 1 , the average local slope of asperities is roughly $10^{\circ}$ with a standard deviation slightly smaller than $10^{\circ}$. This remains acceptable for such an approximation. In that case, deflections of one of the surfaces are linked to the contact pressures by the relation [17]-[18]:

$$
u(\mathbf{x})=\int_{S} U(\mathbf{x}, \xi) p^{c}(\xi) d S
$$

Here, $u(\mathbf{x})$ is the surface deflection at point $\mathbf{x}(x, y)$ of the surface, $U(\mathbf{x}, \xi)$ is the deflection at $\mathbf{x}$ of the surface due to a unit load at $\xi\left(\xi_{x}, \xi_{y}\right)$ while $p^{c}(\xi)$ designates the contact pressure at $\xi$ and $S$ the apparent contact surface.

When solids are elastic and homogeneous, the influence coefficient $U(\mathbf{x}, \xi)$ can be expressed by the method of potentials proposed by Boussinesq [19]:

$$
U(\mathbf{x}, \xi)=\frac{1-\nu^{2}}{\pi E} \frac{1}{\sqrt{\left(x-\xi_{x}\right)^{2}+\left(y-\xi_{y}\right)^{2}}}
$$

where $E$ is the Young's modulus and $\nu$ the Poisson's ratio of the surface under consideration.

Contact pressures must also verify:

$$
\frac{1}{S} \int_{S} p^{c}(\mathbf{x}) d S=\text { Pca }
$$

where Pca is the average applied contact pressure.

From equation (15), we can notice that only surfaces have to be discretized. Computational time is therefore drastically reduced in comparison with clas- 
sical methods like finite element methods, which require the discretization of the entire volume.

If each rough surface is discretized by $\left(n_{x}, n_{y}\right)$ identical cells of dimensions $\left(\Delta_{x}, \Delta_{y}\right)$, the resolution of the elastic deformation problem consist in solving the following system:

$$
\begin{aligned}
& u_{i j}=\sum_{k=0}^{n_{x}-1} \sum_{l=0}^{n_{y}-1} U_{i-k, j-l} p_{k l}^{c}, \quad\left(0 \leq i \leq n_{x}, 0 \leq j \leq n_{y}\right) \\
& \frac{1}{n_{x} n_{y}} \sum_{i=0}^{n_{x}-1} \sum_{j=0}^{n_{y}-1} p_{i j}^{c}=\text { Pca } \\
& u_{i j}=\delta_{0}-h_{i j}, \quad(i, j) \in \Omega_{c} \\
& p_{i j}^{c}>0, \quad(i, j) \in \Omega_{c} \\
& u_{i j} \geq \delta_{0}-h_{i j}, \quad(i, j) \notin \Omega_{c} \\
& p_{i j}^{c}=0, \quad(i, j) \notin \Omega_{c}
\end{aligned}
$$

where $u_{i j}$ is the surface deflection at node $(i, j), p_{i j}^{c}$ is the uniform contact pressure acting on the cell centered at node $(i, j), U_{i-k, j-l}$ are the influence coefficients, $\Omega_{c}$ is the set of grid nodes that are in contact, $\delta_{0}$ is the rigid body displacement of the two solids and $h_{i j}$ is the aperture at node $(i, j)$ between undeformed surfaces.

The problem is solved with an iterative scheme based on a conjugate gradient technique [20]. The numerical cost of the discrete convolution of equation (18a) is high due to the multi-summation. If a Fourier transform is performed on this equation, the convolution product is replaced by a multiplication:

$$
\tilde{u}_{i j}=-\tilde{U}_{i j} \tilde{p}_{i j}^{c}, \quad\left(0 \leq i \leq n_{x}, 0 \leq j \leq n_{y}\right)
$$

where $\tilde{a}$ stand for the Fourier transform of $a$. The use of a fast Fourier transform allows to reduce the number of operation and thus computational times.

The solution also takes into account plastic deformations. The elasto-plastic model is directly applied to contact pressures. If the local pressure is higher than a limit pressure, a plastic criterion is applied. In this work, materials are assumed to follow a pure plastic behavior so that, local pressures are bounded by the hardness $\mathcal{H}$ of the softer material.

\subsection{Transport models}

\subsubsection{Microscopic viscous flow}

Assuming the flow to be isothermal, stationary, incompressible and at small Reynolds number (creeping flow), the viscous flow through the contact, at the 
scale of the roughness, can be described by the Stokes model:

$$
\begin{aligned}
& -\nabla p+\mu \nabla^{2} \mathbf{v}=0 \\
& \nabla \cdot \mathbf{v}=0 \\
& \mathbf{v} \cdot \mathbf{n}=0, \text { at the solid wall }
\end{aligned}
$$

$\mathbf{v}$ being the fluid velocity, $p$ the fluid pressure and $\mu$ its dynamic viscosity.

If we assume that the aperture field $h(x, y)$ is varying slowly (i.e. that the slopes $\alpha$ of asperities are small), the momentum equation (20a) can be reduced to the simpler Reynolds equation [21]. This can be shown by introducing the dimensionless quantities $x^{*}=\frac{x}{l^{0}}, y^{*}=\frac{y}{l^{0}}, z^{*}=\frac{z}{h^{0}}, v_{x, y}^{*}=\frac{v_{x, y}}{u^{0}}$ and $v_{z}^{*}=\frac{v_{z}}{w^{0}}$, where $l^{0}$ and $h^{0}$ are characteristic lengths in $(x, y)$ - and $z$-directions respectively (see fig. 5), while $u^{0}$ and $w^{0}$ are characteristic magnitudes of velocity in $(x, y)$ - and $z$-directions respectively. With such scales, the mass conservation equation (eq. (20b)) can be rewritten as:

$$
\frac{\partial v_{x}^{*}}{\partial x^{*}}+\frac{\partial v_{y}^{*}}{\partial y^{*}}+\frac{l^{0} w^{0}}{h^{0} u^{0}} \frac{\partial v_{z}^{*}}{\partial z^{*}}=0
$$

To ensure that the last term has the same order of magnitude as the two first terms, we assume that:

$$
w^{0}=\frac{h^{0}}{l^{0}} u^{0}
$$

If we consider that slopes of asperities are small, i.e. $h^{0}<<l^{0}$, the dimensionless form of equation (20a) can be reduced to:

$$
\begin{aligned}
& -\frac{\partial p^{*}}{\partial \lambda^{*}}+\frac{\partial^{2} v_{\lambda}^{*}}{\partial z^{* 2}}=0, \lambda^{*}=\left(x^{*}, y^{*}\right) \\
& -\frac{\partial p^{*}}{\partial z^{*}} \approx 0
\end{aligned}
$$

where we define $p^{*}$ as $p^{*}=\frac{\left(h^{0}\right)^{2}}{\mu l^{0} u^{0}} p$ in order to keep all terms of the same order of magnitude in the momemtum equation. Equation (23b) means that $p$ is $z$-independent. Thus, the momentum equation (20a) can be reduced to the $2 \mathrm{D}$ form $\left(\mathbf{v}\left(v_{x}, v_{y}\right)\right)$ :

$$
\nabla p(x, y)=\mu \frac{\partial^{2} \mathbf{v}}{\partial z^{2}}
$$

leading to a local Poiseuille flow in the $x$ - and $y$-directions. If $\lambda$ and $\chi$ are two orthogonal directions $(\lambda, \chi=x, y)$, integrating this parabolic velocity profile on a section of height $h(x, y)$ and width $d \chi$ yields the viscous flow rate $d Q_{v \lambda}$ 
in the $\lambda$-direction. The viscous flow rate $d q_{v \lambda}$ per unit length $d \chi$ is given by:

$$
q_{v \lambda}=\frac{d Q_{v \lambda}(x, y)}{d \chi}=-\frac{h^{3}(x, y)}{12 \mu} \frac{\partial p(x, y)}{\partial \lambda},(\lambda, \chi)=(x, y) \text { and } \lambda \neq \chi
$$

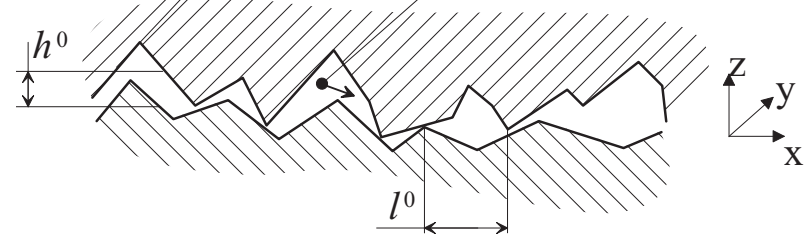

Fig. 5. Rough contact -

Equation (25) is known as the Reynolds equation. Integrating the continuity equation (20b) over $z$ and using the no-slip condition, we show that the viscous flux $\mathbf{q}_{v}\left(q_{v x}, q_{v y}\right)$ is a divergence free vector. Under these circumstances, if $\beta$ designates the fluid phase and $\sigma$ the solid phase (i.e. the contact areas) in the $x-y$ plane (see fig. 6), the viscous flow problem, now reduced from $3 \mathrm{D}$ to $2 \mathrm{D}$, can be expressed as:

$$
\begin{aligned}
& \mathbf{q}_{v}=-\frac{h^{3}}{12 \mu} \nabla p \text { in } \beta \\
& \nabla \cdot \mathbf{q}_{v}=0 \text { in } \beta \\
& \mathbf{q}_{v} \cdot \mathbf{n}=0 \text { on } C_{\beta \sigma}
\end{aligned}
$$

$\mathbf{n}$ being the unit normal vector to the contours $C_{\beta \sigma}$ of the contact areas.

S

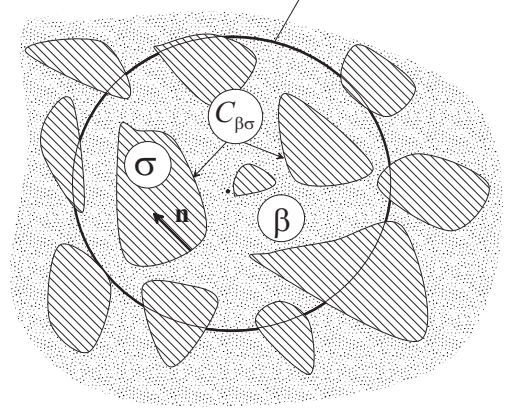

Fig. 6. Two-phase medium -

\subsubsection{Diffusion at the micro-scale}

With the assumptions of a stationary and isothermal mass transfer, the 3D diffusive process can be described by the classical Fick's law:

$$
\mathbf{j}=-\mathcal{D} \nabla c
$$




$$
\nabla \cdot \mathbf{j}=0
$$

$\nabla c \cdot \mathbf{n}=0$ at the solid wall

where $\mathbf{j}$ is the diffusive flux, $c$ the species concentration which diffuses through the contact and $\mathcal{D}$ the molecular diffusion coefficient. As for viscous effects, using dimensionless variables $j_{x, y}^{*}=\frac{j_{x, y}}{j_{1}^{0}}, j_{z}^{*}=\frac{j_{z}}{j_{2}^{0}}$, where $j_{1}^{0}$ and $j_{2}^{0}$ are characteristic magnitudes of flux (see fig. 5) in $(x, y)$ - and $z$-directions respectively, it can be assumed from equation (27b) that:

$$
j_{2}^{0}=\frac{h^{0}}{l^{0}} j_{1}^{0}
$$

Considering again the hypothesis $h^{0}<<l^{0}$, the Fick's law (27a) can be reduced to:

$$
\begin{aligned}
& j_{\lambda}^{*}=-\frac{\partial c^{*}}{\partial \lambda^{*}}, \lambda^{*}=\left(x^{*}, y^{*}\right) \\
& \frac{\partial c^{*}}{\partial z^{*}} \approx 0
\end{aligned}
$$

where $c^{*}$ is defined by $c^{*}=\frac{\mathcal{D}}{j_{1}^{0} l^{0}} c$. Equation (29b) means that $c$ is $z$-independent. The diffusive problem is also made two-dimensional and Fick's law becomes:

$$
\mathbf{j}=-\mathcal{D} \nabla c(x, y)
$$

As previously, by integrating equation (30) in a section of height $h(x, y)$ and width $d y$ (or $d x$ ), we obtain the local diffusive flow rate $d Q_{d x}$ (or $d Q_{d y}$ ) and the equivalent lineic flow rate $q_{d x}$ (or $q_{d y}$ ):

$$
q_{d \lambda}=\frac{d Q_{d \lambda}(x, y)}{d \chi}=-\mathcal{D} h(x, y) \frac{\partial c(x, y)}{\partial \lambda},(\lambda, \chi)=(x, y) \text { and } \lambda \neq \chi
$$

Integrating the continuity equation (27b) over $z$ and making use of the boundary condition (27c) shows as for viscous flow that the diffusive flux vector $\mathbf{q}_{d}\left(q_{d x}, q_{d y}\right)$ is divergence free.

Finally, the diffusive problem in a two-phase medium (see fig. 6) can be rewritten as follows:

$$
\begin{aligned}
& \mathbf{q}_{d}=-\mathcal{D} h(x, y) \nabla c \text { in } \beta \\
& \nabla \cdot \mathbf{q}_{d}=0 \text { in } \beta \\
& \mathbf{q}_{d} \cdot \mathbf{n}=0 \text { on } C_{\beta \sigma}
\end{aligned}
$$

\subsubsection{Transport properties of the rough contact}

Previous models describe viscous and diffusive flows at the microscale, i.e. at the scale of asperities. The two problems being similar, they can be rewritten 
in a generic form:

$$
\begin{aligned}
& \mathbf{q}=-k \nabla \omega \text { in } \beta \\
& \nabla \cdot \mathbf{q}=0 \text { in } \beta \\
& \mathbf{n .} k \nabla \omega=0 \text { on } C_{\beta \sigma}
\end{aligned}
$$

with $k=\frac{h^{3}}{12 \mu}$ and $\omega=p$ for viscous flow and $k=\mathcal{D} h(x, y)$ and $\omega=c$ for diffusion. We now need to relate the macroscopic flow rate per width unit, at the scale of surface element under consideration in this work, to the macroscopic driving force (i.e. the macroscopic pressure gradient or species gradient). To do so, we can employ a formalism identical to volume averaging [22]. This is performed by averaging equations (33) over $S$ (see fig. 6) making use of two averaging operators defined as:

$$
\langle\varphi\rangle=\frac{1}{S} \int_{S_{\beta}} \varphi d S=\frac{1}{S_{\beta}+S_{c}} \int_{S_{\beta}} \varphi d S
$$

and

$$
\langle\varphi\rangle^{\beta}=\frac{1}{S_{\beta}} \int_{S_{\beta}} \varphi d S
$$

along with the averaging theorem:

$$
\langle\nabla \varphi\rangle=\nabla\langle\varphi\rangle+\frac{1}{S} \int_{C_{\beta \sigma}} \mathbf{n} \varphi d S
$$

Without providing details (see [22] for the method and [23]-[24] for developments on similar problems), the generic macroscopic equation takes the form:

$$
\langle\mathbf{q}\rangle=-\mathbf{H} \cdot \nabla\langle\omega\rangle^{\beta}
$$

In equation (35), the tensor $\mathbf{H}$ is given by:

$$
\mathbf{H}=\langle k(\mathbf{I}+\nabla \mathbf{b})\rangle
$$

where $\mathbf{b}$ is the solution of the closure problem that is written as:

$$
\begin{aligned}
& \nabla \cdot(k \nabla \mathbf{b})=-\nabla \widetilde{k} \text { in } \beta \\
& -\mathbf{n} \cdot \nabla \mathbf{b}=\mathbf{n} \text { on } \mathrm{C}_{\beta \sigma} \\
& \langle\mathbf{b}\rangle=0 \\
& \mathbf{b}\left(\mathbf{x}+\mathbf{r}_{i}\right)=\mathbf{b}(\mathbf{x})
\end{aligned}
$$

with $\widetilde{k}=k-\langle k\rangle$ and $\mathbf{r}_{i}$ the surface element dimension in the $i^{\text {th }}$ direction. This surface element is supposed to be representative of a periodic infinite structure. 
The transmissivity tensor $\mathbf{K}$ (viscous flow) allows to express the lineic flow rate at the scale of the surface element as:

$$
\left\langle\mathbf{q}_{v}\right\rangle=-\frac{\mathbf{K}}{\mu} \cdot \nabla\langle p\rangle^{\beta}
$$

where $\mathbf{K}=\mathbf{H}$ with $k=\frac{h^{3}}{12}$ in (36); $\mathbf{K}$ has the dimension of length to the cube.

In the same way, the effective diffusivity tensor $\mathbf{D}$ leads to the expression of the lineic flow rate by diffusion at the scale of the element of surface as:

$$
\left\langle\mathbf{q}_{d}\right\rangle=-\mathcal{D D} \cdot \nabla\langle c\rangle^{\beta}
$$

where $\mathbf{D}=\mathbf{H}$ with $k=h$ in (36). Note that $\mathbf{D}$ has the dimension of length.

As can be seen from the definition of $\mathbf{K}$ and $\mathbf{D}$ and from equations (37), these two tensors are intrinsic, i.e. they depend only on the structure of the aperture field $h(x, y)$ and on the contact areas.

Equations (37) were derived while considering a continuous aperture field. However, if roughness is measured on a set of $n_{x} \mathrm{x} n_{y}$ points so that the surface is represented by $n_{x} \times n_{y}$ cells, each of them having a constant aperture $h_{i}$, the discrete form of the closure problem is:

$$
\begin{aligned}
& i^{\text {th }} \text { cell: } \nabla \cdot \nabla \mathbf{b}_{i}=0 \\
& i j \text { interface: } \mathbf{n} \cdot k_{i}\left(\nabla \mathbf{b}_{i}+\mathbf{I}\right)=\mathbf{n} \cdot k_{j}\left(\nabla \mathbf{b}_{j}+\mathbf{I}\right) \\
& i j \text { interface: } \mathbf{b}_{i}=\mathbf{b}_{j} \\
& j^{\text {th }} \text { cell: } \nabla \cdot \nabla \mathbf{b}_{j}=0 \\
& \sum_{i} b_{i}=0
\end{aligned}
$$

Equations (40b) and (40c) allow the continuity of $\mathbf{n} . \mathbf{q}$ and $\omega$ respectively, between each cell. Moreover, at the scale of the surface element, $\mathbf{b}$ is assumed to be periodic. Methods to approximate $\mathbf{H}$ could be employed to estimate, at least, its diagonal component in the direction of $\nabla\langle\omega\rangle^{\beta}[25]$ using the effective medium theory [26]-[27] or a stochastic approach [28]. To avoid other approximations than the Reynolds one, the problem (40) is solved here in a similar way to that proposed for instance by Brown [29] using a finite volume scheme that is second order in space [30]. The computational grid exactly corresponds to that used for surface measurement or generation. Due to the discontinuity of $k$ (and hence of $\nabla \mathbf{b}$ ) at the interface between two adjacent grid blocks, a second order approximation of the flux of $\mathbf{b}$ on this interface requires the use of the harmonic mean of $k_{i}$ and $k_{j}$ as proposed for the problem of heat conduction in heterogeneous materials [31]. The linear system obtained from this 
finite volume discretization is solved using a preconditionned conjugate gradient algorithm [32]. This provides the $\mathbf{b}$ field that is further used to compute $\mathbf{H}$ according to equation (36). It should be noticed that since $\mathbf{K}$ and $\mathbf{D}$ are related to two very distinct averages ( $h^{3}$ and $h$ respectively) of the aperture field, the comparison on $\mathbf{K}$ and $\mathbf{D}$ computed on real and synthesized surfaces provide two efficient discriminating tests of the validity of the representation of the self-affine fractal surfaces under consideration.

\subsection{Algorithm}

The computational algorithm used to determine transport properties of a rough contact is shown in figure 7 . It is initiated with a set of $n_{x} \mathrm{x} n_{y}$ points $z(x, y)$ describing a representative surface element either of the real or synthesized surface. The aperture field $h(x, y)$ resulting from deformation of the initial surface $z(x, y)$ is computed with the elasto-plastic deformation model as described above. Intrinsic transmissivity and diffusivity tensors, $\mathbf{K}$ and $\mathbf{D}$ respectively, are computed using the same numerical procedure. Distinction between viscous and diffusive effects is performed by the surface preparation module. The percolation module allows to remove all non percolating clusters, i.e. non contact areas not connected to surface edges.

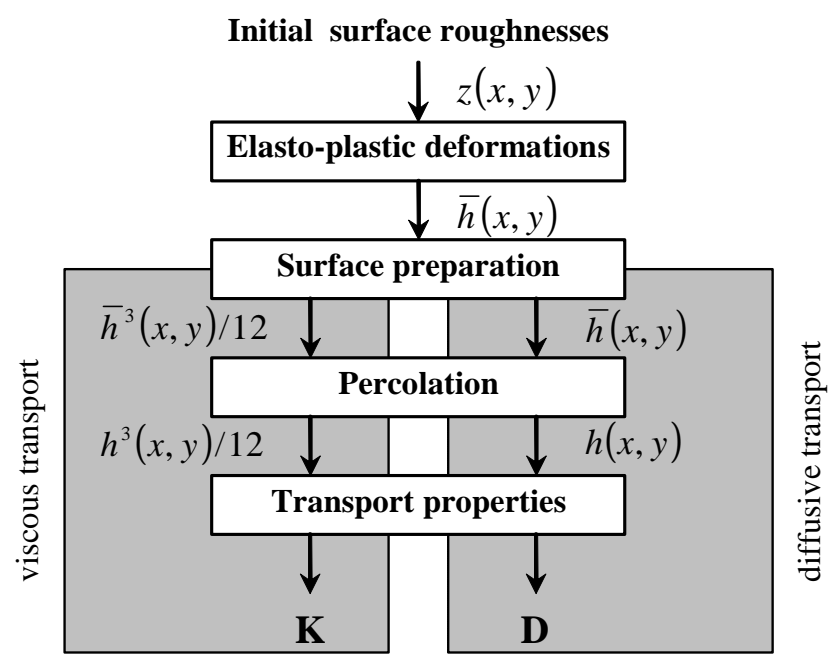

Fig. 7. Computational scheme -

\section{Results and discussions}

In this section, we consider the contact between one of the rough surfaces presented in section 2 and a perfectly rigid and smooth plane. Results obtained 
from lapped and sand-blasted real and synthesized surfaces are compared in order to validate the use of synthetic self-affine fractal surfaces. Two aspects are investigated: the distribution of contact spots on the one hand and viscous and diffusive transport properties of the contact on the other hand. Computations were performed with the mechanical properties of stainless steel (see tab. 2) for several average contact pressures Pca ranging from 7 to $600 \mathrm{MPa}$.

Several self-affine fractal surfaces were synthesized using the same roughness parameters but changing the initial random sequence $a_{i j}$. Thus, for each kind of texture (lapped and sand-blasted), four different surfaces were synthesized from the power spectral densities of the real surfaces considered in section 2 .

Table 2

Mechanical properties of stainless steel -

\begin{tabular}{lll}
\hline Young's modulus & Poisson's ratio & Hardness \\
$E$ & $\nu$ & $\mathcal{H}$ \\
\hline $210000 \mathrm{MPa}$ & 0.3 & $1800 \mathrm{MPa}$ \\
\hline
\end{tabular}

\subsection{Effective contact areas}

In figure 8, we have represented the ratio of the true contact area $S_{c}$ to the nominal surface area $S=L_{x} L_{y}$ versus the average contact pressure Pca. The linear dependence of $S_{c} / S$ on Pca is confirmed and an excellent agreement is observed between real and synthesized surfaces. However, it must be noticed that the successful comparison between the effective contact areas is not sufficiently discriminating since, as can be seen from the graphs in figure 8 , lapped and sand-blasted surfaces exhibit identical variations of $S / S_{c}$ versus Pca, although these surfaces have roughness parameters that are significantly different. To be more conclusive, a comparison of the distribution of local contacts is necessary.

In figures 9 and 10, we have reported the mean, $\mathrm{m}$, and the standard deviation, $\sigma$, of the distribution of contact spot areas versus the contact pressure Pca for lapped and sand-blasted surfaces respectively. For lapped surfaces, we can observe that all the synthesized surfaces have a similar behavior, while for sand-blasted surfaces, a more significant dispersion between the four realizations is observed. As noticed in section 2, lapped surfaces differ from sand-blasted ones in the relative power of low frequencies. Contrary to lapped surfaces, the dominant pattern of sand-blasted surfaces is determined by the longer wavelengths close to the scale of the surface element under consideration. Thus, while changing the initial random sequence $a_{i j}$, the texture of sand-blasted surfaces is more affected than that of lapped surfaces, that is why a significant scatter is observed among synthesized sand-blasted surfaces 


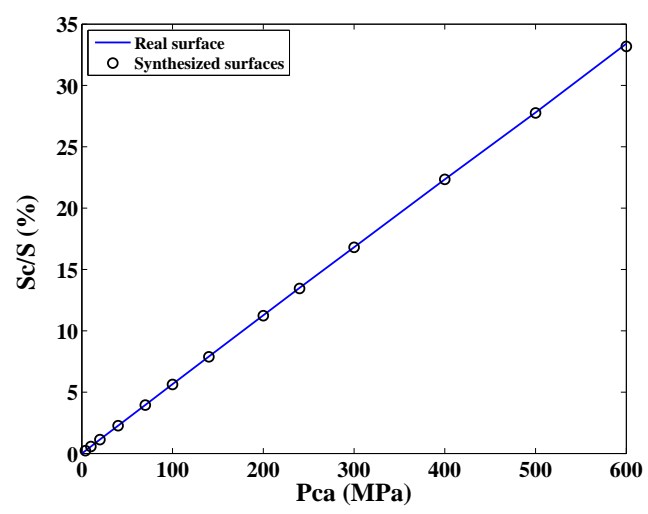

- Lapped surface -

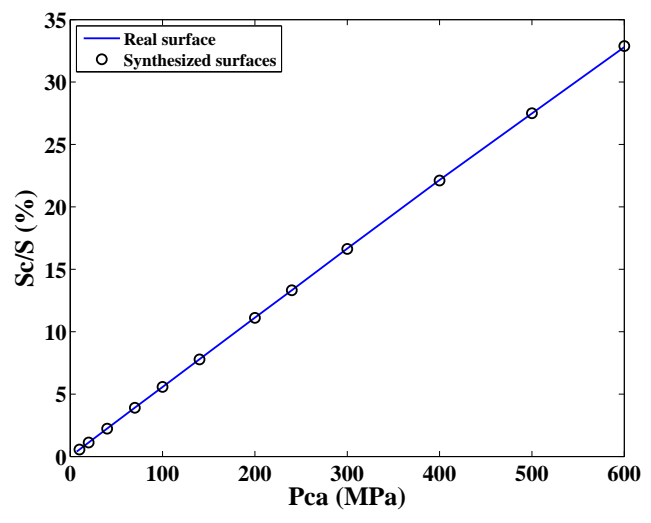

- Sand-blasted surface -

Fig. 8. Relative contact area $S_{c} / S$ versus average contact pressure Pca -

and not among synthesized lapped surfaces.

As can be seen in figure 9, a good agreement between real and synthesized surfaces is observed on the mean, $\mathrm{m}$, of the contact spot areas since relative deviations between real and synthesized lapped surfaces are smaller than $10 \%$. The comparison of the standard deviations $\sigma$ shows that this parameter is more sensitive since relative deviations are about $30 \%$.

Deviations between the mean of the real sand-blasted surface and the average value of the corresponding synthesized surfaces (figure 10) can reach 20\%, which remains fully consistent in comparison to the relative dispersion on the four realizations of synthesized surfaces (about 15\%). Results concerning the standard deviation $\sigma$ show a good agreement between real and synthesized surfaces since results obtained on the real surface fall well within the range of those obtained on synthesized surfaces.

\subsection{Effective transport properties}

Because of machining process, surfaces under investigation here are expected to be isotropic in the $x-y$ plane, featuring spherical $\mathbf{K}$ and $\mathbf{D}$ tensors, i.e. $\mathbf{K}=\mathrm{K} \mathbf{I}$ and $\mathbf{D}=\mathrm{DI}$. Our numerical results confirm this property (at least for lapped surface; see discussions below). In fact, off-diagonal terms for the two types of surfaces were, at least, two orders of magnitude smaller than diagonal terms. As a consequence, our discussion is focused on the diagonal terms of $\mathbf{K}$ and $\mathbf{D}$. Figure 11 shows the log-log plots of the diagonal terms of $\mathbf{K}$ and $\mathbf{D}$ of the real and synthesized lapped surfaces versus the average contact pressure Pca. This figure clearly indicates that the real surface is perfectly isotropic at the scale of investigation. All synthesized surfaces have the same transport properties, except at high contact pressure Pca where some dispersion can be observed. At low Pca, the number of percolating clusters, i.e. paths where the 


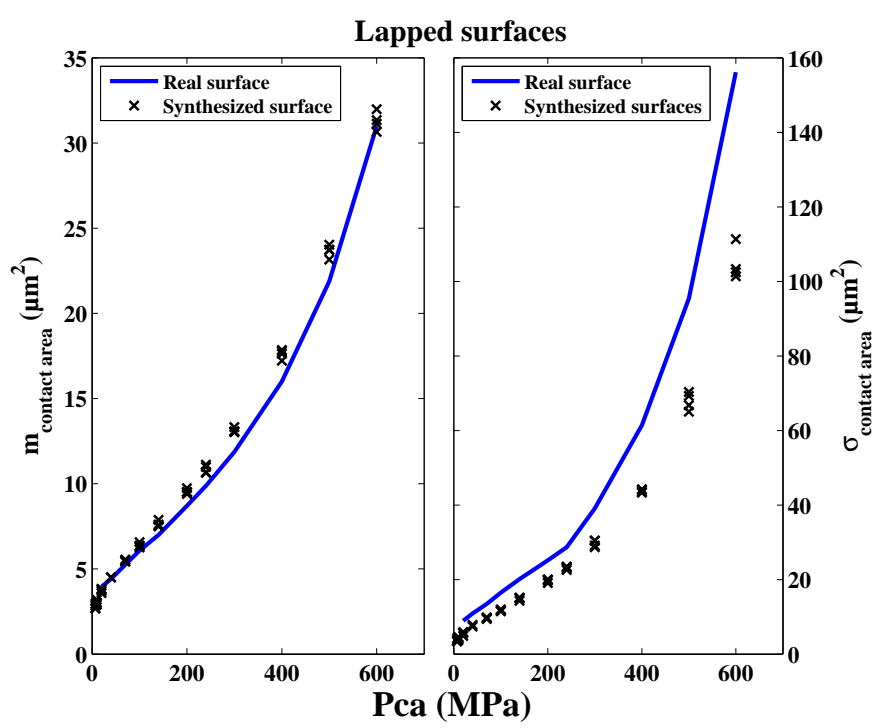

Fig. 9. Mean, $\mathrm{m}$, and standard deviation, $\sigma$, of the distribution of contact spot areas of lapped surfaces -

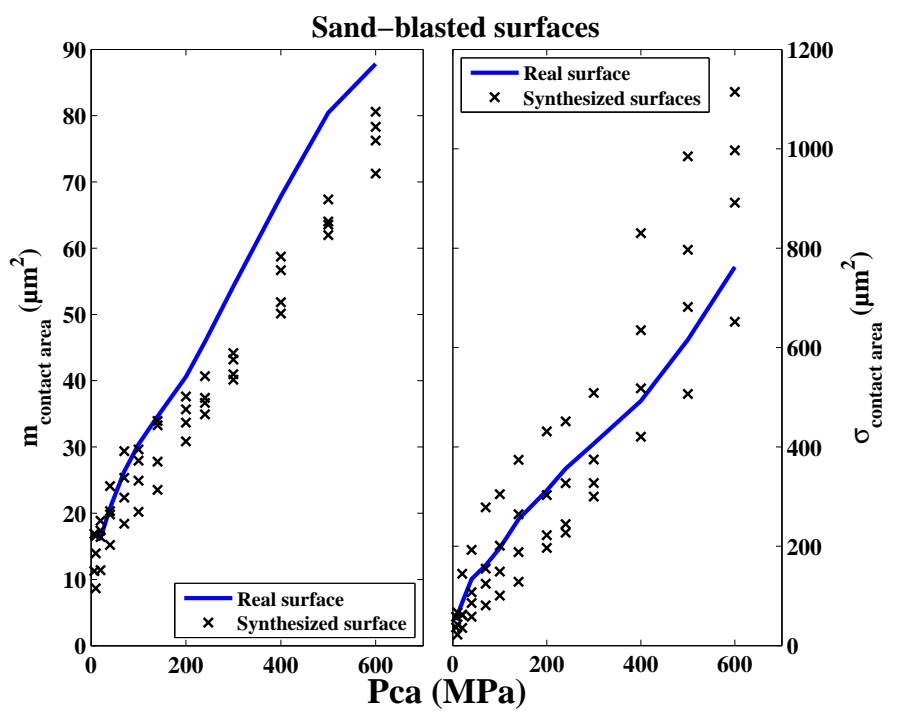

Fig. 10. Mean, m, and standard deviation, $\sigma$, of the distribution of contact spot areas of sand-blasted surfaces -

fluid can flow, is large, explaining why all surfaces are statistically identical. When increasing Pca, the number of percolating clusters decreases, leading to scattered values of $\mathrm{K}$ and $\mathrm{D}$. Moreover, transport properties rapidly decrease with the increasing of the contact pressure which confirms that tightening makes the contact less percolating. Whereas experimental results obtained on turned surfaces indicate that both $\mathrm{K}$ and $\mathrm{D}$ depend on Pca according to a power law [33], effective transport properties of self-affine fractal surfaces exhibit a more complex behavior. It must be noticed that the range of variation of $\mathrm{K}$ is much wider than that of $\mathrm{D}$, and this is due to the fact that this 
later quantity is related to the aperture field $h$ while the former is related to $h^{3}$. For both $\mathrm{K}$ and $\mathrm{D}$, an excellent agreement is obtained between real and synthesized surfaces. Whatever Pca, relative deviations remain smaller than $7 \%$ on the estimation of $\mathrm{D}$. It can reach $20 \%$ on the estimation of $\mathrm{K}$, which is small compared to the range of variation of $\mathrm{K}$ over roughly three orders of magnitude for the range of Pca under consideration.

In figure 12, we have reported the diagonal terms of $\mathbf{K}$ and $\mathbf{D}$ estimated on the real and synthesized sand-blasted surfaces. In contrast to lapped surfaces, diagonal terms are significantly different as evidenced by results on the real surface. The same behavior is observed on each of the four realizations. For each surface, at a given value of Pca, relative deviations of diagonal terms with respect to their average value increase with contact pressure Pca. As for lapped surfaces, this is due to the number of percolating clusters which decreases when Pca increases. These deviations can reach $20 \%$ for diffusivity and $45 \%$ for transmissivity. Anisotropy of the transport properties is a result of a size effect, as already discussed above for the distribution of contact spot areas. Since the fractal character of this surface remains at a scale equal to the size $L_{x}, L_{y}$ of the surface element under investigation, dispersion is expected on its behavior that must be analyzed on average over several realizations. However, as for lapped surfaces, a very good agreement between computed transport properties is obtained between real and synthesized sand-blasted surfaces. Along with results on effective contact areas, this validates the use of synthesized surfaces for the estimation of both the effective contact and the transport properties of a rough contact between self-affine fractal surfaces.

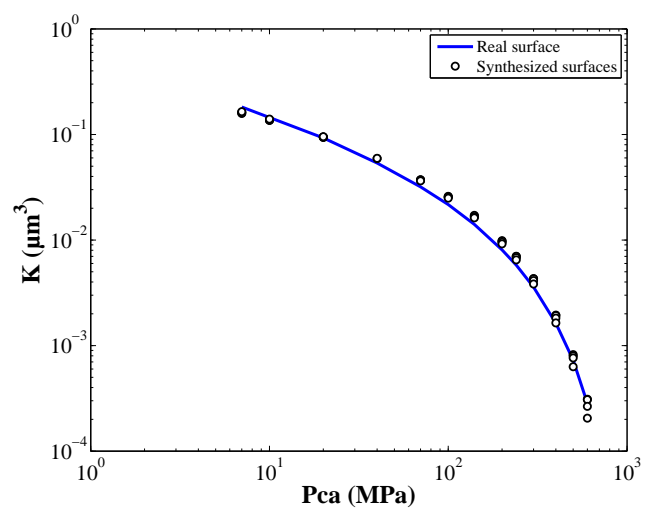

- Transmissivity -

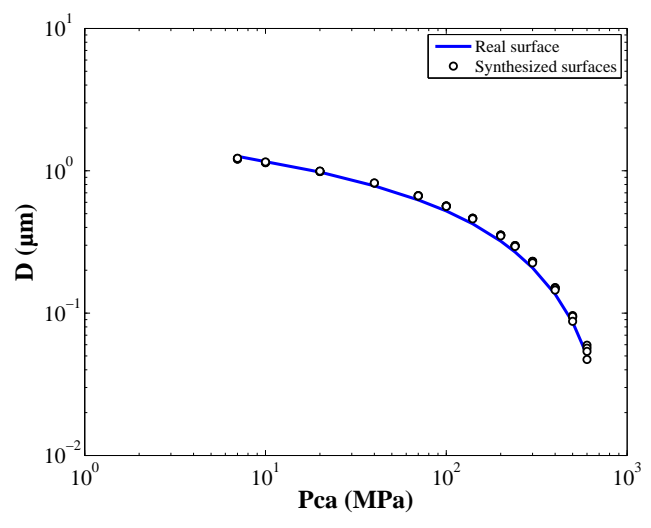

- Diffusivity -

Fig. 11. Transport properties of the real and synthesized lapped surfaces - 


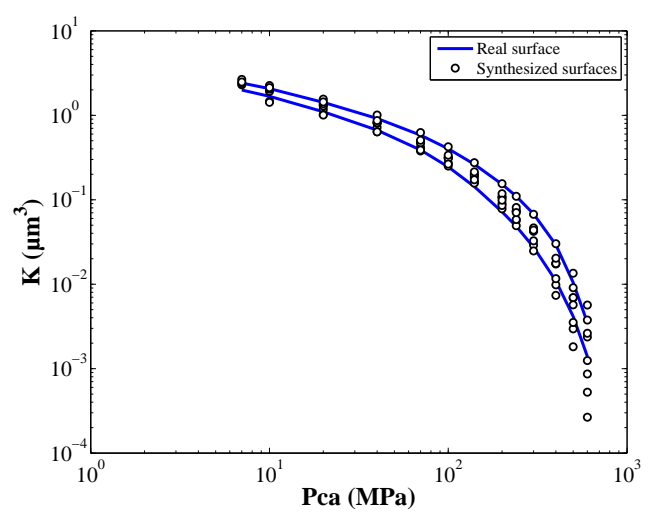

- Transmissivity -

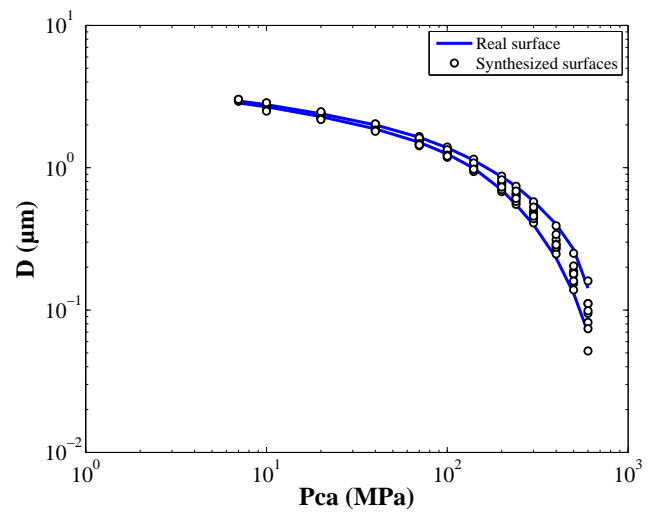

- Diffusivity -

Fig. 12. Transport properties of the real and synthesized sand-blasted surfaces -

\section{Conclusions}

A global and deterministic approach for computing both deformation under load and transport properties of a rough contact from surface textures has been presented. The study was carried out on a class of rough surfaces exhibiting fractal properties. The fractal nature of textures produced by lapping and sand-blasting was confirmed from direct measurements. These two kinds of surface textures differ in their spectral densities: sand-blasted surfaces exhibit fractal properties at every scale investigated whereas lapped surfaces exhibit fractal properties only for frequencies higher than a cut-off frequency $\omega_{\min }$. Textures of sand-blasted surfaces are consequently less regular than those of lapped surfaces since they contain longer wavelengths.

Equivalent self-affine fractal surfaces were synthesized with the same fractal properties as the real surfaces and a thorough comparison of contact areas on the one hand and of transport properties on the other, was performed between real and synthesized surfaces. Due to the existence of the cut-off frequency, the study on lapped surfaces shows that when synthesizing several surfaces with the same roughness parameters, results are almost identical. This is not true for sand-blasted surfaces and significant dispersions are observed between results obtained from four realizations of synthesized surfaces. For the same reasons, at the scale of the surfaces investigated in this work, transport properties of lapped surfaces are isotropic whereas due to a size effect, transport properties of sand-blasted surfaces are anisotropic.

The main result of this work is twofold. First, distributions of contact areas of the synthesized surfaces were shown to be in good agreement with those of the real surfaces. Secondly, a very good agreement was obtained between real and synthesized surfaces during the estimation of viscous and diffusive transport properties, for both lapped and sand-blasted surfaces. This represents a set 
of very discriminating comparisons leading to the conclusion that synthesized self affine surfaces can be used to describe contact mechanics and contact transport properties through a contact between rough surfaces produced by a random machining process.

\section{Acknowledgements}

Financial support from EDF R\&D as well as from CNES, SNECMA and CNRS are gratefully acknowledged.

\section{References}

[1] B. Bhushan, Contact mechanics of rough surfaces in tribology: Multiple asperity contact, Tribology Letters 4 (1998) 1-35.

[2] B. Bhushan, Surface Roughness Analysis and Measurement Techniques, Vol. 1, edited by B. Bhushan, CRC Press, Boca Raton, 2001.

[3] C. Marie, D. Lasseux, H. Zahouani, P. Sainsot, An integrated approach to characterize liquid leakage through metal contact seal, European Journal Mech. and Env. Eng. 48 (2) (2003) 81-86.

[4] S. Ganti, B. Bhushan, Generalized fractal analysis and its applications to engineering surfaces, Wear 180 (1995) 17-34.

[5] L. He, J. Zhu, The fractal character of processed metal surfaces, Wear 208 (1997) 17-24.

[6] R. Jahn, H. Truckenbrodt, A simple fractal analysis method of the surface roughness, Journal of Materials Processing Technology 145 (2004) 40-45.

[7] A. Majumdar, B. Bhushan, Role of fractal geometry in roughness characterization and contact mechanics of surfaces, Journal of Tribology 112 (1990) 205-216.

[8] A. Majumdar, C. Tien, Fractal characterization and simulation of rough surfaces, Wear 136 (1990) 313-327.

[9] H. Peitgen, D. Saupe, The Science of Fractal Images, Springer-Verlag, New York, 1988.

[10] B. Mandelbrot, Self-affine fractals and fractal dimension, Physica Scripta 32 (1985) 257-260.

[11] B. Persson, O. Albohr, U. Tartaglino, A. Volokitin, E. Tosatti, On the nature of surface roughness with application to contact mechanics, sealing, rubber friction and adhesion, J. Phys.: Condens. Matter 17 (2005) R1-R62.

[12] J. Greenwood, J. Williamson, Contact of nominally flat surfaces, Proceedings of the Royal Society of London A295 (1966) 300-319. 
[13] W. Chang, I. Etsion, D. Bogy, An elastic-plastic model for the contact of rough surfaces, Journal of Tribology 109 (1987) 257-263.

[14] P. Nayak, Random process model of rough surfaces in plastic contact, Wear 26 (1973) 305-333.

[15] A. Bush, R. Gibson, G. Keogh, Strongly anisotropic rough surfaces, Journal of Lubrication Technology 101 (1979) 15-20.

[16] A. Bush, R. Gibson, T. Thomas, The elastic contact of a rough surface, Wear 35 (1975) 87-111.

[17] C. Mayeur, P. Sainsot, L. Flamand, A numerical elastoplastic model for rough contact, Journal of Tribology 117 (1995) 422-429.

[18] P. Sainsot, C. Jacq, D. Nélias, A numerical model for elastoplastic rough contact, CMES 3 (4) (2002) 497-506.

[19] K. Johnson, Contact Mechanics, Cambridge University Press, Cambridge, 1985.

[20] I. Polonsky, L. Keer, A numerical method for solving rough contact problems based on the multi-level multi-summation and conjugate gradient techniques, Wear 231 (1999) 206-219.

[21] R. Zimmerman, G. Bodvarsson, Hydraulic conductivity of rock fractures, Transport in Porous Media 23 (1996) 1-30.

[22] S. Whitaker, The Method of Volume Averaging, Theory and Application of Transport in Porous Media, Kluwer Academic Publishers, 1999.

[23] M. Prat, F. Plouraboué, N. Letalleur, Averaged Reynolds equation for flow between rough surfaces in sliding motion, Transport in Porous Media 48 (2002) 291-313.

[24] M. Quintard, S. Whitaker, Ecoulement monophasique en milieux poreux : Effets des hétérogénéités locales, Journal de Mécanique Théorique et Appliquée 6 (1987) 691-726.

[25] P. M. Adler, J. F. Thovert, Fractures and fracture networks, Kluwer Acad., Dordrecht, 1999.

[26] J. B. Walsh, S. R. Brown, W. B. Durham, Effective media theory with spatial correlation for flow in a fracture, Journal of Geophysical Research 102 (1997) $22587-22594$.

[27] G. Drazer, J. Koplik, Transport in rough self-affine fractures, Physical Review E 66 (2) (2002) 026303/1-026303/16.

[28] J. Inoue, H. Sugita, Fourth-order approximation of fluid flow through roughwalled rock fracture, Water Resources Research 39 (8) (2003) SBH1 1-SBH1 10 .

[29] S. Brown, Transport of fluid and electric current through a single fracture, Journal of Geophysical Research 94 (1989) 9429-9438. 
[30] R. Peyret, T. D. Taylor, Computational Methods for Fluid Flow, SpringerVerlag, New York, 1983.

[31] S. V. Patankar, Numerical Heat Transfer and Fluid Flow, Hemisphere Pub. Corp., Washington, 1980.

[32] G. Meurant, The Lanczos and Conjugate Gradient Algorithms: From Theory to Finite Precision Computation, SIAM, Philadelphia, 2006.

[33] C. Marie, D. Lasseux, Experimental leak-rate measurement through a static metal seal, Journal of Fluids Engineering 129 (6) (2007) 799-805. 